\title{
Exploring different constraint-based modelings for program verification
}

\author{
Hélène Collavizza and Michel Rueher \\ Université de Nice-Sophia-Antipolis - I3S/CNRS \\ 930, route des Colles - B.P. 145, 06903 Sophia-Antipolis, France. \\ $\{$ helen, rueher\}@polytech.unice.fr
}

\begin{abstract}
Recently, constraint-programming techniques have been used to generate test data and to verify the conformity of a program with its specification. Constraint generated for these tasks may involve integer ranging on all machine-integers, thus, the constraint-based modeling of the program and its specification is a critical issue. In this paper we investigate different models. We show that a straightforward translation of a program and its specification in a system of guarded constraints is ineffective. We outline the key role of Boolean abstractions and explore different search strategies on standard benchmarks.
\end{abstract}

\section{Introduction}

Constraint programming techniques have been used to generate test data (e.g., $[6,13])$ and to develop efficient model checking tools (e.g. [10,4]). SAT based model checking platforms have been able to scale and perform well due to many advances in SAT solvers [11]. Recently, constraint-programming techniques have also been used to verify the conformity of a program with its specification $[3,8]$.

To establish the conformity between a program and its specification we have to demonstrate that the union of the constraints derived from the program and the negation of the constraints derived from its specification is inconsistent. Roughly speaking, pruning techniques -that reduce the domain of the variablesare combined with search and enumeration heuristics to demonstrate that this constraint system has no solutions.

Experimentations reported in [8] demonstrate that constraint techniques can be used to handle non-trivial academic examples. However, we are far from the state where this techniques can be used automatically on real applications. Modeling is a critical issue, even on quite small programs. That's why we investigate different models in this paper.

The framework we explore in this paper can be considered as a specific instance of SMT solvers ${ }^{1}$.

\footnotetext{
${ }^{1}$ For short, a Satisfiability Modulo Theories (SMT) problem consists in deciding the satisfiability of ground first-order formulas with respect to background theories such as the theory of equality, of the integer or real numbers, of arrays, and so on $[5,12$, 1]
} 
The different models and search strategies we did experiment with, showed that a straightforward translation of a program and its specification in a system of guarded constraints is ineffective ${ }^{2}$. These experimentations also clearly outlined the key role of appropriate Boolean abstractions.

The verification of the conformity between a program and its specification is highly dependant on the programming language and the specification language. In this paper, we restrict ourseleves to Java programs and JML specifications (for "Java Modeling Language" see www.cs.iastate.edu/ leavens/JML).

To illustrate the advantages and limits of the models and search strategies we introduce, we will use the following examples:

- S1 (see figure 1) : This is a very simple example of a Java program and its JML specification : it returns 1 if $i<j$ and 10 otherwise. S1 will help us to understand how to introduce boolean abstraction. For each model, we will give the complete constraint system for $\mathrm{S} 1$.

- S2 : This is also a very simple example derived from S1. The only difference with $\mathrm{S} 1$ is that it returns calculus on input variables instead of constant values, i.e., it returns $i+j$ when $\mathrm{S} 1$ returns 1 (line 5 in figure 1 ) and $2 * i$ when $\mathrm{S} 1$ returns 10 (line 6 in figure 1). The main idea is to evaluate the impact of arithmetic during the resolution process.

- Tritype : This is a famous example in test case generation and program verification. This program takes three numbers that must be positive. These numbers represent the lengths of three sides of a triangle. It returns 4 if the input is not a triangle, 3 if it is an equilateral triangle, 2 if it is isosceles and 1 if it is a scalene triangle.

- Tri-perimeter: This program has the same control part than the tritype program. It returns -1 if the three inputs are not the lengths of triangle sides, else it returns the perimeter of the triangle. The specification returns $i+j+k$ while the program returns either $3 * i$ or $2 * i+j, 2 * i+k, 2 * j+i$ or $i+j+k$.

The program and the specification of tritype and tri-perimeter can be found at www.polytech.unice.fr ${ }^{\sim}$ rueher/annex_tritype_triperimeter.pdf.

All the java programs of the examples in this paper conform to their JML specifications. This corresponds to the much difficult case problem, since the search must be complete. Indeed, detection of non-conformity is much easier in practice (it stops when the first solution is found) even if the difficulty from a theoretical point of view is the same.

The rest of this paper is organised as follows. Section 2 recalls some basics on the translation process we have implemented to generate the constraint systems. Section 3 details the different models we propose whereas section 4 introduces different solving strategies. Section 5 describes the experimental results and section 6 discusses some critical issues.

\footnotetext{
${ }^{2}$ A full translation in Boolean constraints is also ineffective as soon as numeric expression occurs in the program or in the specification. Indeed, in this case we need to translate each integer operation into bit-vector calculus. Thus, even SMT solvers like SMT-CBMC [2] which use specialized solver for bit-vector calculus fail to solve some trivial problems.
} 


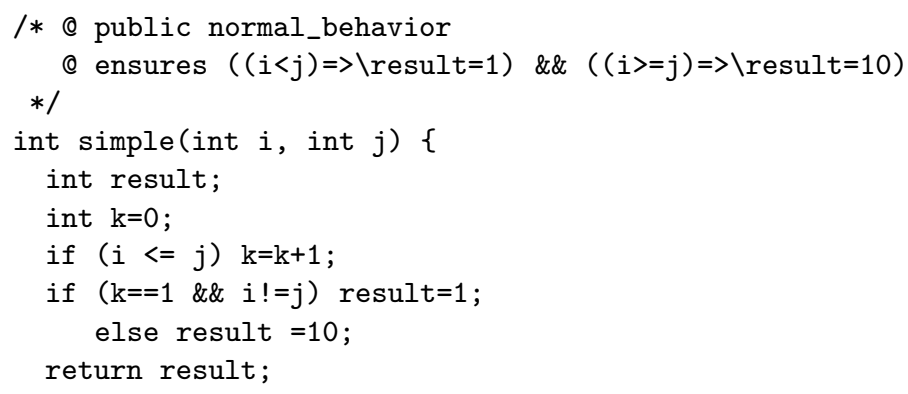

Fig. 1. S1 example : JML specification and Java program

\section{Translation of a program and its specification into constraints}

This section recalls basic techniques for translating a Java program and its JML specification into a set of constraints.

For the sake of simplicity, we only consider here a very restricted form of Java and JML programs. For the JML specification, we restrict ourselves to normal behaviour, i.e., we do not consider exception such as overflow. We also assume that the JML specification contains an \ensures statement, a logical expression defining the post-condition. It may also contain a \requires statement defining the pre-condition. Likewise, we only consider Java program where all variables are integers and we assume that functions have only one return statement. Finally, we do not detail here the process for handling loops. Interested readers can find details on the way we handle loops and several JML statements such as \forall statement in [8].

\subsection{Translation process}

We only recall here the basics which are required to understand this paper. More details on SSA form can be found in [9].

Translating the program into a set of constraints We first transform the program into its SSA "Single State Assignment" form [9]: for each new definition of a program variable, we introduce a fresh variable. In order to manage control instructions, we use $\phi$-functions for if then else statements.

Basic statements Each assignment var = value is translated as a constraint $v a r_{i}=$ value where $i$ is the current number of definition of variable var. For example, the following piece of code $x=x+1 ; y=x * y ; x=x+y$; is translated as the set of constraints: $\left\{x_{1}=x_{0}+1, y_{1}=x_{1} * y_{0}, x_{2}=x_{1} * y_{1}\right\}$. 
Conditional execution flow Conditional execution flows are translated into guarded constraints. Guarded constraints are conditional constraints whose evaluation depends upon other constraints. $C_{0} \rightarrow C_{1}$ denotes a guarded constraint where $C_{0}$ and $C_{1}$ are conjunctions of basic constraints. Relation $C_{0} \rightarrow C_{1}$ states that constraints $C_{1}$ have to be added to the current constraint store when the solver can prove that constraints $C_{0}$ hold. More precisely, let $C_{0}$ be a boolean expression and $C_{1}$ a set of constraints, the guarded constraint $C_{0} \rightarrow C_{1}$ behaves as follows:

- When the solver can prove that $C_{0}$ is true, then constraints $C_{1}$ are added to the store of constraints;

- When the solver can prove that $C_{0}$ is false, then the guarded constraint is just discarded;

- When the solver can neither prove that $C_{0}$ is true, nor prove that $C_{0}$ is false, that is when not enough variables of $C_{0}$ are instantiated, then the guarded constraint is suspended.

The solver tries to prove that the guard $C_{0}$ of a suspended constraint holds whenever the domain of some variable occurring in $C_{0}$ has been reduced. Of course, some guarded constraints may never become active.

One major difficulty with guarded constraints is that nothing can be done before the solver can demonstrate that the condition is either true or false. Let us consider a very simple piece of code:

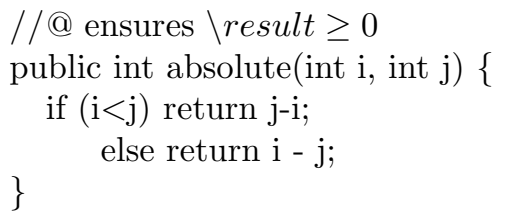

This code is translated into the following set of constraints:

$\{i<j \rightarrow r=j-i, !(i<j) \rightarrow r=i-j, r<0\}$

A standard CSP solver cannot achieve any pruning on this system since nothing is known about $i$ and $j$. So a very costly enumeration process is started: the inconsistency is only detected when the domain of $i$ and $j$ are reduced to one value.

That's why we introduce Boolean variables and handled in a better way guarded constraints (see part 3.2).

The If then statement For the sake of clarity, we only focus on the assignment of a single variable. Trivially, the same process could be applied individually for each variable appearing in a block with many variable assignments.

Let us consider the statement $\mathrm{S}$ : if (cond) \{var=val\}. Assume that var has already been defined $p$ times before this statement. $\mathrm{S}$ is translated into the following set of guarded constraints where $S S A(s)$ denotes the constraint corresponding to the SSA form of the basic statement $s$. 
if part : $\quad \operatorname{SSA}($ cond $) \rightarrow \operatorname{var}_{p+1}=\mathrm{SSA}($ val $)$

else part $: \quad \mathrm{SSA}($ !cond $) \rightarrow \quad \operatorname{var}_{p+1}=\operatorname{var}_{p}$

The else part is crucial to ensure that the $v_{a r}+1$ fresh variable will not remain uninstantiated in the corresponding CSP.

The If then else statement Let us consider the statement $S$ : if (cond) $\left\{\mathrm{v}=x_{1} ; \mathrm{v}=x_{2} ; \ldots ; \mathrm{v}=x_{q} ;\right\}$ else $\left\{\mathrm{v}=y_{1} ; \mathrm{v}=y_{2} ; \ldots ; \mathrm{v}=y_{r} ;\right\}$. Assume that $v$ has already been defined $p$ times before this statement and assume that $q<r$. Since $v$ has not the same number of definitions in the if part and the else part, we need to introduce a guarded constraint to take the place of the $\phi$ function. So, $S$ is translated into the following set of guarded constraints :

// if part

$\operatorname{SSA}($ cond $) \rightarrow\left(v_{p+1}=\operatorname{SSA}\left(x_{1}\right)\right) \&\left(v_{p+2}=\operatorname{SSA}\left(x_{2}\right)\right) \& \ldots \&\left(v_{p+q}=\operatorname{SSA}\left(x_{q}\right)\right)$

// else part

$\mathrm{SSA}($ !cond $) \rightarrow\left(v_{p+1}=\operatorname{SSA}\left(y_{1}\right)\right) \&\left(v_{p+2}=\operatorname{SSA}\left(y_{2}\right)\right) \& \ldots \&\left(v_{p+r}=\operatorname{SSA}\left(y_{r}\right)\right)$

$/ / \phi$ function

$\mathrm{SSA}($ cond $) \rightarrow\left(v_{p+q+1}=v_{p+q}\right) \&\left(v_{p+q+2}=v_{p+q}\right) \& \ldots \&\left(v_{p+r}=v_{p+q}\right)$

Remark: if $q>r$ the same principle is applied and the guarded constraints of the $\phi$ function are guarded by $S S A(\neg$ cond). If $\mathrm{q}=\mathrm{r}$ then no $\phi$ function is required. Figure 2 gives an example of translation of an overlapped if then else.

1

2

3

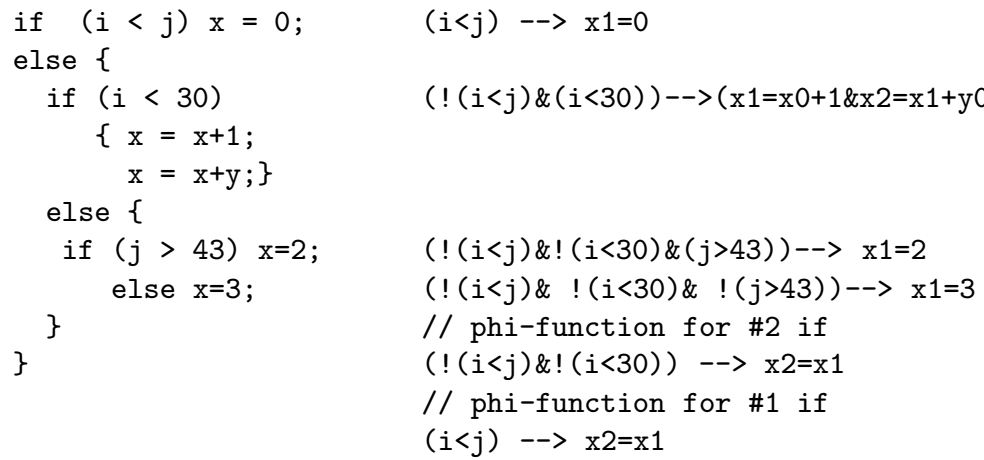

Fig. 2. example of if then else translation

Specification translation The JML specification we handle is decomposed into two parts : the \requires statement and the \ensures statement. The $\backslash$ requires statement is a logical expression on input variables and the \ensures statement is a logical expression on input variables and \result, which denotes in JML the value returned by the method. We translate the JML specification in the following way : 
- each logical expression is translated in the corresponding constraint,

- the \result JML variable is associated to a special variable of the CSP named result which establishes the link between the program and the specification,

- we add the constraints issued from the \requires statement,

- we add the negation of constraints issued from the \ensures statement.

\subsection{Characteristics of a CSP for software validation}

We define a CSP for software validation as a tuple formed with a set of integer variables, a set of boolean variables (possibly empty) used to improve performance of guarded constraints propagation, a set of guarded constraints issued from the program and the specification and an abstraction table which gives the correspondence between the boolean abstract variables and the integer expressions. This is detailed in figure 3 .

1. Variables

- INT_VAR : set of finite variables with domain [min,max]

- BOOL_VAR : set of boolean variables with domain $[0,1]$

- result $\in$ INT_VAR : the variable which makes the link between the program (Java return statement) and the specification ( \result JML statement)

2. Constraints

- PROG_CONST : set of guarded constraints from the program

- REQUIRE_CONST : set of guarded constraints from the JML \requires statement

- ENSURE_CONST : set of guarded constraints from the negation of the JML $\backslash$ ensures statement

3. Abstraction table

- SEMANTICS $\left(b_{i}\right)$ provides the finite domain constraint that is modelled by the boolean abstract variable $b_{i}$.

Fig. 3. CSP for software validation

\section{$3 \quad$ Modeling issues}

We present here different models from the less abstract one to the most abstract one that we studied during our experiments. All the models are illustrated on the $S 1$ example of figure 1 . To help the reading, in the successive figures for $S 1$ example, we start by ' $*$ ' the lines which have changed from one model to next. However, when solving the CSP, all the models are also evaluated on the two more significant benchmarks tritype and tri-perimeter. 


\subsection{INT_CSP: a model without boolean abstraction}

In this model, we do not introduce any boolean variable : the guarded constraints are couples $(\mathrm{g}, \mathrm{c})$ where $\mathrm{g}$ and $\mathrm{c}$ are expressions on integer variables. Figure 4 illustrates this model on example $S 1$.

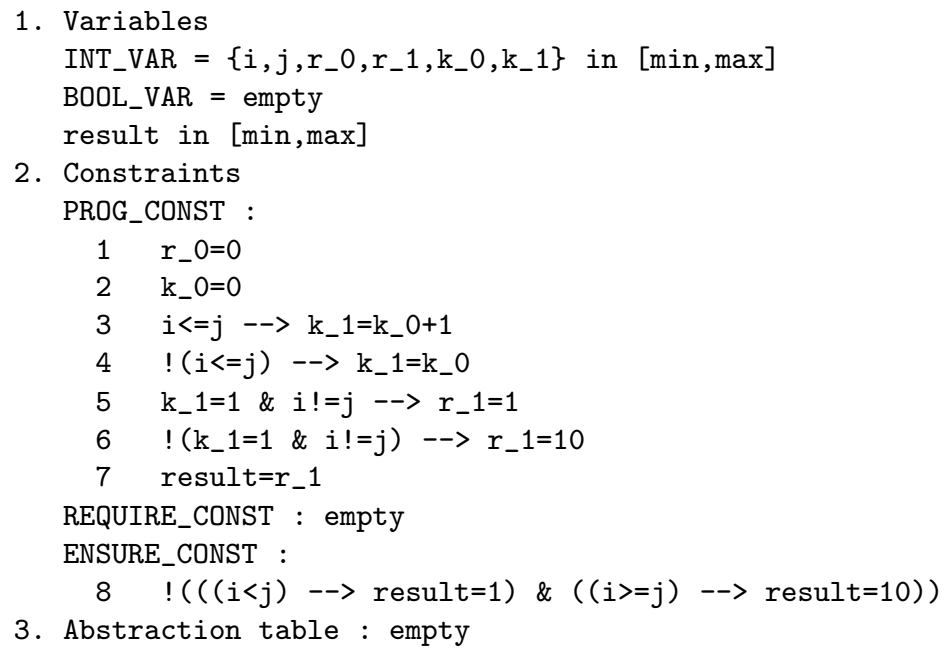

Fig. 4. S1 example : INT_CSP

\subsection{HYBRID_CSP_1: using Boolean abstraction for the program guards}

In this model, we introduce a boolean variable for each guard expression involved in the constraints of the program. The advantage is that the enumeration process begins with boolean variables and so guards are evaluated first. In this way, a constraint $c$ can be posted even if the integer variables involved in its guard are not instantiated. The introduction of boolean entails a non standard processing of guarded constraints. For instance, consider the guarded constraint $i<j \rightarrow$ $k_{1}=k_{0}+1$ and assume that the boolean variable $b_{0}$ is associated to the guard $i<j$. When $b_{0}$ is set to true, both constraints $i<j$ and $k_{1}=k_{0}+1$ are added to the constraint store.

Figure 5 shows this model on the $S 1$ example. With respect to figure 4 we have introduced two boolean variables, g_0 for the guard of constraints 3 and 4 and g_1 for the guard of constraints 5 and 6 .

\subsection{HYBRID_CSP_2: using Boolean abstraction for expressions appearing in several guards}

One drawback of the previous model is that it looses too much semantics : it doesn't take into account that the same logical expressions may be involved 
1. Variables

INT_VAR $=\left\{i, j, r_{-} 0, r_{-} 1, k_{-} 0, k_{-} 1\right\}$ in $[\min , \max ]$

* $\quad$ BOOL_VAR $=\left\{g_{-} 0, g_{-} 1\right\}$ in $[0,1]$

result in [min,max]

2. Constraints

PROG_CONST :

$1 r_{-} 0=0$

$2 \mathrm{k}_{-} 0=0$

* 3 g_o $\rightarrow \mathrm{k}_{-} 1=\mathrm{k}_{-} 0+1$

* 4 ! g_o $\rightarrow \mathrm{k}_{-} 1=\mathrm{k}_{-} 0$

* 5 g_1 $\rightarrow r_{-} 1=1$

* 6 !g_1 - $\quad r_{-} 1=10$

7 result $=r_{-} 1$

REQUIRE_CONST : empty

ENSURE_CONST :

$8 \quad !(((i<j)-\rightarrow$ result $=1) \&((i>=j)-->$ result $=10))$

3. Abstraction table

* $\operatorname{SEMANTICS}\left(g_{-} 0\right)=i<=j$

* $\operatorname{SEMANTICS}\left(g_{-} 1\right)=k_{-} 1=1 \& i !=j$

Fig. 5. S1 example : HYBRID_CSP_1

in distinct guards. That's why we introduce a boolean variable for each subexpression which appears several times in the guards of the program constraints or in the specification constraints in the model HYBRID_CSP_2.

In the previous modeling of $S 1$ example (see figure 5), the sub-expression $i<j$ appears both in guard g_0 and in the specification constraints. So we introduced a new boolean variable to abstract this expression (see figure 6).

\subsection{HYBRID_CSP_3: adding boolean abstraction for expressions involving the variable result}

In order to have a better link between the program and the specification, we extend the HYBRID_CSP_2 model by introducing a boolean abstraction for each expression on the result variable. This variable is part both of the program and the specification and so it can be helpful to cut some branches during the resolution process. Since result can take only one value, we add a constraint which states that only one abstract variable can be true at the same time.

For example $S 1$, we introduced two boolean variables in the model of figure 6 : one for the expression result=1 and the other for the expression result=10; the link between the possible values of variable result is done by constraint 7 in figure 7 .

\subsection{BOOL_CSP: Boolean model}

In this model, we introduce a boolean variable for each expression in the program and the specification. This is the model which is used by SAT solvers when the 
1. Variables

INT_VAR $=\left\{i, j, r_{-} 0, r_{-} 1, k_{-} 0, k_{-} 1\right\}$ in [min, max]

* $\quad$ BOOL_VAR $=\left\{g_{-} 0, g_{-} 1, g_{-} 2\right\}$ in $[0,1]$

result in [min,max]

2. Constraints

PROG_CONST

$1 r_{-} 0=0$

$2 \mathrm{k}_{-} \mathrm{O}=0$

* 3 (g_ol|g_1) $-->\mathrm{k}_{-} 1=\mathrm{k}_{-} 0+1$

* 4 ! (g_ol|g_1) $-\rightarrow \mathrm{k}_{-} 1=\mathrm{k}_{-} 0$

* 5 g_2 $-\rightarrow r_{-} 1=1$

* 6 !g_2 --> $r_{-} 1=10$

7 result $=r_{-} 1$

REQUIRE_CONST : empty

ENSURE_CONST

$8 !\left(\left(g_{-} 0-->\right.\right.$ result $\left.=1\right)$ \& $\left(! g_{-} 0-->\right.$ result $\left.\left.\left.=10\right)\right)\right)$

3. Abstraction table

* $\operatorname{SEMANTICS}\left(g_{-} 0\right)=i<j$

* $\operatorname{SEMANTICS}\left(g_{-} 1\right)=i=j$

* $\operatorname{SEMANTICS}\left(\mathrm{g}_{-} 2\right)=\mathrm{k}_{-} 1=1 \&$ ! $\mathrm{g}_{-} 1$

Fig. 6. S1 example : HYBRID_CSP_2

expressions do not contain any arithmetic expression. If they do, basic arithmetic operations must also be modelled with boolean constraints.

\section{Solving the CSP}

We have explored various strategies for solving hybrid CSP with boolean and integer variables. These strategies are closely related to the abstraction models we presented in the previous section. We operated with JSolver4Verif: it is a Java version of Ilog solver ${ }^{3}$ with specific propagation rules based on congruence techniques. However, on all the examples contained in this paper the performances of JSolver are very similar to the one of Solver.

\subsection{Solving an integer CSP}

In order to solve INT_CSP we only have to enable a search on the CSP. If a solution is found, then it is an error test case (a data that satisfies the constraints of the program and of the negation of its specification); otherwise the program is conform to its specification.

\footnotetext{
${ }^{3}$ See http://www.ilog.com/products/cp/.
} 
1. Variables

INT_VAR $=\left\{i, j, r_{-} 0, r_{-} 1, k_{-} 0, k_{-} 1 \backslash\right\}$ in $[\min , \max ]$

* $\quad$ BOOL_VAR $=\left\{g_{-} 0, g_{-} 1, g_{-} 2, b_{-} 0, b_{-} 1\right\}$ in $[0,1]$

result in [min,max]

2. Constraints

PROG_CONST :

$1 r_{-} 0=0$

$2 \mathrm{k}_{-} 0=0$

3 (g_ol|g_1) $\rightarrow \mathrm{g}_{-} 1=\mathrm{k}_{-} 0+1$

4 ! (g_ol|g_1) $->\mathrm{k}_{-} 1=\mathrm{k}_{-} 0$

* 5 g_2 $\rightarrow->b_{-} 0$

* 6 !g_2 $->b_{-} 1$

* 7 b_0 + b_1 = 1

REQUIRE_CONST : empty

ENSURE_CONST :

$8 !\left(\left(g_{-} 0->b_{-} 0\right)\right.$ \& $\left.\left(! g_{-} 0->b_{-} 1\right)\right)$

3. Abstraction table

* SEMANTICS[g_0] $=i<j$

$* \quad$ SEMANTICS [g_1] $=i=j$

* SEMANTICS[g_2] $=\mathrm{k}_{-} 1=1$ \& $! \mathrm{g}_{-} 1$

* SEMANTICS[b_0] $=$ result $=1$

* SEMANTICS [b_1] = result $=10$

Fig. 7. S1 example : HYBRID_CSP_3

\subsection{Solving a hybrid CSP using a CSP solver}

We show here two strategies for solving hybrid CSP for models HYBRID_CSP_1, HYBRID_CSP_2 and HYBRID_CSP_3:

- Strategy 1 : searching for all solutions.

Roughly speaking, this strategy consists into searching a solution to the hybrid CSP and then to construct an integer CSP which has the semantics of the current solution of the hybrid CSP. This process is detailed in figure 9. The goal of this strategy is to take advantage of of the forward and backward propagation process on guarded constraints.

- Strategy 2 : enumerating on boolean variables only

This is the same algorithm as Strategy 1 except that in line 1 of figure 9 we start the search by enumerating on boolean variables only (i.e. on B_VAR variables only).

This strategy is mandatory when we use models HYBRID_CSP_2 or HYBRID_CSP_3. In these models, some boolean variables depend from other boolean variables and do not appear directly in the constraints of the program and the specification. So they can remain uninstantiated during the search on the HYBRID_CSP. 
1. Variables

INT_VAR $=\left\{i, j, r_{-} 0, r_{-} 1, k_{-} 0, k_{-} 1\right\}$ in [min, max]

$* \quad$ BOOL_VAR $=\left\{g_{-} 0, g_{-} 1, g_{-} 2, b_{-} 0, b_{-} 1, b_{-} 2, \ldots, b_{-} 11\right\}$ in $[0,1]$

result in [min,max]

2. Constraints

PROG_CONST :

* 1 b_0

* 2 b_1

* 3 (g_ol|g_1) $-->b_{-} 2$

* 4 ! (g_ol|g_1) $-\rightarrow$ b_3

* 5 g_2 $-->b_{-} 4$

* 6 ! g_2 $-\rightarrow b_{-} 5$

* 7 b_2 + b_3 = 1

* 8 b_4 + b_5 = 1

REQUIRE_CONST : empty

ENSURE_CONST :

* 9 ! ( (g_0 --> b_4) \& (!g_0 $\left.\left.->b_{-} 5\right)\right)$

3. Abstraction table

$\operatorname{SEMANTICS}\left(g_{-} 0\right)=i<j$

$\operatorname{SEMANTICS}\left(g_{-} 1\right) \quad=i=j$

$\operatorname{SEMANTICS}\left(\mathrm{g}_{-} 2\right)=\mathrm{k}_{-} 1=1 \quad \& \quad \mathrm{~g} \_1$

* $\operatorname{SEMANTICS}\left(\mathrm{b}_{-} 0\right)=r_{-} 0=0$

* $\operatorname{SEMANTICS}\left(\mathrm{b} \_2\right)=\mathrm{k}_{-} 1=\mathrm{k}_{-} 0+1$

* $\quad$ SEMANTICS $\left(b_{-} 4\right)=$ result $=1$

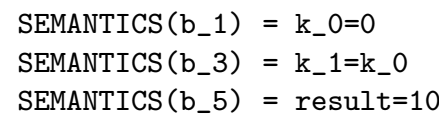

Fig. 8. S1 example : BOOL_CSP

\section{Experimental results}

\subsection{Experimental results}

In this section we evaluate the various models and strategies on the $S 1$ and $S 2$ programs and also validate our conclusions on the two more realistic examples tritype and tri-perimeter.

In each table, we consider signed integers and we give the time performance according to the number of bits they are coded with. Since our purpose is to compare different modeling issues, we use a time out limit of ten minuts (denoted '-' in the tables). Nevertheless, we give the solving time required for the maximum size of integers in some cases (in italic). bool denotes the number of booleans solutions, that's to say the number of finite domain CSP which are generated, and which have to be disproved to demonstrate the conformity between the program and its specification.

The constraint systems for these different programs have been generated automatically. Primary boolean expressions are stored on the fly in a hash map and each expression is replaced with a boolean variable if it is used more than once, depending on the model. All experiments were performed on a Processor Intel Core 2 Duo E6400 (2,13 GHz, 1G memory). 


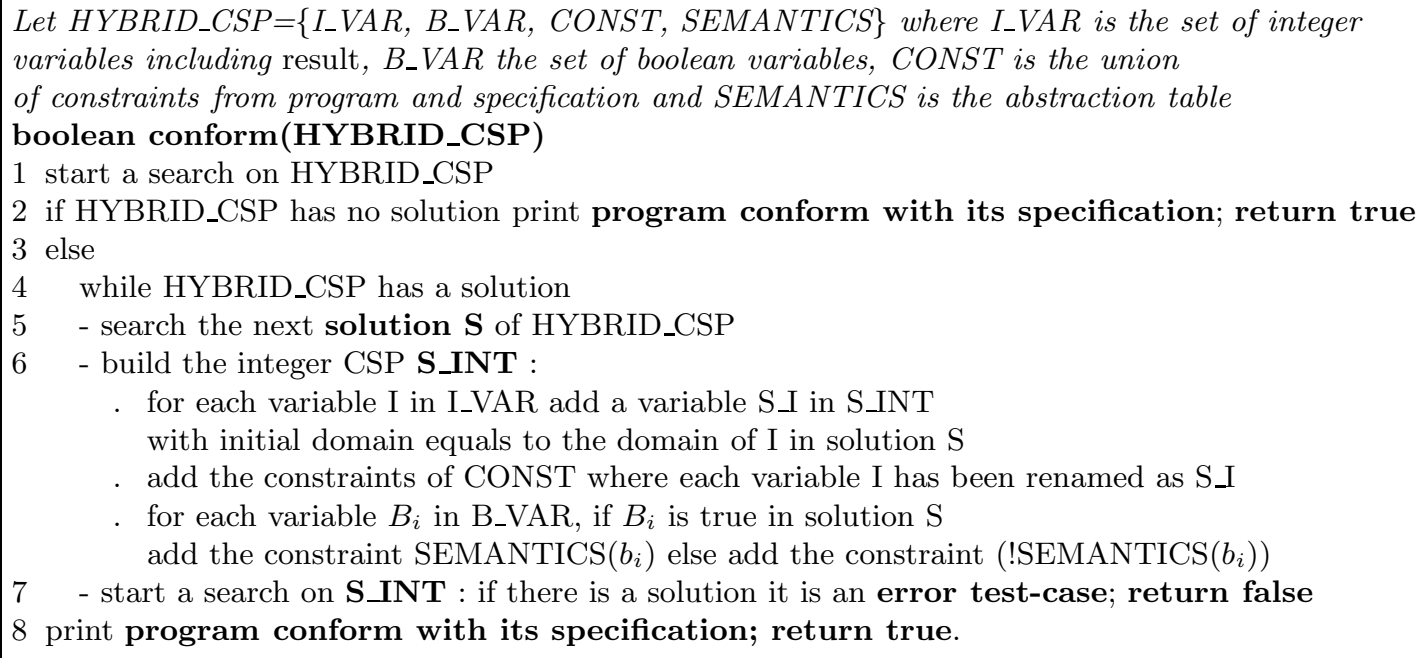

Fig. 9. Strategy 1 : solving an hybrid CSP

\subsection{INT_CSP model}

As mentioned in subsection 3.2, the INT_CSP model cannot achieve any filtering as long as the integer variables involved in the guards are not instantiated. So, the search process is very slow: table 1 shows that even for the very simple examples, the INT_CSP model cannot be solved for integers coded on 32 bits.

\begin{tabular}{|l|c|c|c|c|}
\hline \# bit & S1 & S2 & tritype & tri-perimeter \\
\hline 8 & $0.577 \mathrm{~s}$ & $0.766 \mathrm{~s}$ & $66.582 \mathrm{~s}$ & $406.27 \mathrm{~s}$ \\
10 & $5.422 \mathrm{~s}$ & $9.255 \mathrm{~s}$ & - & - \\
16 & $21663.778 \mathrm{~s}$ (6 hours) & - & - & - \\
32 & - & - & - & - \\
\hline
\end{tabular}

Table 1. CSP_INT solving

\subsection{Hybrid CSP models}

Table 2 provides the results for $S 1$ and tritype programs.

In these programs, the returned value is a constant so the HYBRID_CSP_3 is not relevant. Indeed, in this case there is little interest to introduce a boolean abstraction for each expression on the result variable.

Searching all solutions (strategy 1 ) is rather inefficient with the HYBRID_CSP_1 model. Strategy 2 (searching only boolean solutions) is clearly better both with HYBRID_CSP_1 and HYBRID_CSP_2. 
An essential observation is that strategy 1 searches for all the solutions, that is to say, even for solutions which differ on integer variables but are equals for boolean variables. As said before, we evaluated this strategy because the inverse propagation of guarded constraints may eliminate values for boolean variables on some problems. Indeed, for a guarded constraint $(g, c)$, if $c$ is proved false due to other constraints, then the negation of $g$ is added to the constraint store; this information may cut some branches for other guarded constraints which share guard $g$.

The difference between the results for the various models highlights that introducing boolean variables is a key issue when these variables are shared by many constraints.

\begin{tabular}{|l|c|c|c|c|c|c|}
\hline \multicolumn{7}{|c|}{ S1 and tritype } \\
\hline \# bit & $\begin{array}{c}\text { HYBRID_CSP_1 } \\
\text { strategy 1 }\end{array}$ & $\begin{array}{c}\text { HYBRID_CSP_1 } \\
\text { strategy 2 }\end{array}$ & \multicolumn{2}{c|}{$\begin{array}{c}\text { HYRID_CSP_2 } \\
\text { strategy 2 }\end{array}$} \\
\hline & S1 & tritype & S1 & tritype & S1 & tritype \\
\hline 8 & $57.116 \mathrm{~s}$ & - & $0.194 \mathrm{~s}$ & $0.999 \mathrm{~s}$ & $0.182 \mathrm{~s}$ & $1.768 \mathrm{~s}$ \\
& 131072 bool & & 4 bool & 565 bool & 4 bool & 4520 bool \\
\hline 10 & - & - & $0.221 \mathrm{~s}$ & $4.157 \mathrm{~s}$ & $0.186 \mathrm{~s}$ & $1.926 \mathrm{~s}$ \\
& & & 4 bool & 565 bool & 4 bool & 4520 bool \\
\hline 16 & - & - & $0.568 \mathrm{~s}$ & - & $0.221 \mathrm{~s}$ & $8.522 \mathrm{~s}$ \\
& & & 4 bool & & 4 bool & 4520 bool \\
\hline 32 & - & - & - & - & $1520.82 ~ s$ & - \\
& & & & & 4 bool & \\
\hline
\end{tabular}

Table 2. HYBRID_CSP_1 and HYBRID_CSP_2 solving

Table 3 compares the performances of models HYBRID_CSP_2, HYBRID_CSP_3 and BOOL_CSP on the $S 2$ and tri-perimeter examples using strategy 2.

First, let us note that the performances are weaker on these two examples. This is due to the arithmetic operations, which occur in these two examples. Indeed, S2 (resp. tri-perimeter) differs from $S 1$ (resp. tritype) only on the operative part (calculus on inputs instead of constant value).

Another essential observation, is that in model BOOL_CSP we provide a constraint which states that the boolean variables which correspond to several possible values of a single variable cannot be true at the same time. This is a critical point: if we remove this constraint for tri-perimeter with integers coded on 8 bits there are 778240 boolean solutions and it takes 319.028 s to solve the problem.

This kind of information could probably be captured by a a global 'cardinality' or 'atmost' constraint. 


\begin{tabular}{|l|c|c|c|c|c|c|}
\hline \multicolumn{7}{|c|}{ S2 and tri-perimeter } \\
\hline \# bit & HYBRID_CSP_2 & \multicolumn{2}{|c|}{ HYBRID_CSP_3 } & \multicolumn{2}{|c|}{ BOOL_CSP } \\
\hline & S2 & tri-perimeter & S2 & tri-perimeter & S2 & tri-perimeter \\
\hline 8 & $0.477 \mathrm{~s}$ & $15.056 \mathrm{~s}$ & $0.185 \mathrm{~s}$ & $6.57 \mathrm{~s}$ & $0.2 \mathrm{~s}$ & $3.42 \mathrm{~s}$ \\
& 8 bool & 5056 & $4 \mathrm{bool}$ & 22464 bool & 4 bool & $6080 \mathrm{bool}$ \\
\hline 10 & $2.946 \mathrm{~s}$ & - & $0.2 \mathrm{~s}$ & $10.489 \mathrm{~s}$ & $0.286 \mathrm{~s}$ & $3.654 \mathrm{~s}$ \\
& 8 bool & & $4 \mathrm{bool}$ & $22464 \mathrm{bool}$ & $4 \mathrm{bool}$ & $6080 \mathrm{bool}$ \\
\hline 16 & - & - & $0.274 \mathrm{~s}$ & - & $0.292 \mathrm{~s}$ & $4.809 \mathrm{~s}$ \\
& & & $4 \mathrm{bool}$ & & 4 bool & $6080 \mathrm{bool}$ \\
\hline 32 & - & - & $2516.156 \mathrm{~s}$ & - & - & - \\
& & & 4 bool & & & \\
\hline
\end{tabular}

Table 3. HYBRID_CSP_2, HYBRID_CSP_3 and BOOL_CSP solving

\section{Discussion}

Verification and validation are two of the most critical issues in the software engineering process. Numerous techniques, ranging from formal proofs to testing methods have been used during the last years to verify the conformity of a program with its specification. However, the verification of the conformity between a program and its specification remains a difficult task, even for small programs. Our experimentations [8] showed that constraints techniques can be very efficient on some non trivial problems. Performance of CSP techniques behave clearly better than state of art SMT solvers $[8,2]$

In this paper, we did investigated different CSP models on a few simple but non-trivial academic example. As expected, a straightforward translation of a program and its specification in a system of guarded constraints is ineffective, even on very simple example. Boolean abstraction is clearly a critical issue for efficiency. An appropriate Boolean abstraction is an essential support for the search process.

Of course, additional work is required before these techniques can be used on real applications. Further work to try is on the cooperation of CSP and SAT solver as well as new filtering techniques.

When the set of Boolean constraints becomes larger, a collaboration between a SAT solver and CSP solver is probably more appropriate to handle this problems. We have performed some very preliminary experimentation with SAT4J(see www.sat4j.org) and Jsolver4Verif. A technical difficulty concerns the enumeration of all solution by a SAT solver. Indeed, the most efficient SAT solver are not designated to enumerate all issue. Moreover, the transfer to the SAT solver of failure information from CSP solver which is a key issue is far from being obvious.

Lazy approches like the ones used in Barcelogic (see www.barcelogic.org) may also improve the performances. 
Specific filtering techniques ${ }^{4}$ may also drastically improve the refutation of the generated CSP over finite domains. Likewise, linear solvers or difference constraint solvers may be used to check the consistency of constraint defining the semantics of guards.

\section{References}

1. Aït-Kaci H., Berstel B., Junker U., Leconte M., Podelski A. : Satisfiability Modulo Structures as Constraint Satisfaction : An Introduction. Procs of JFLA 2007, 8 pages.

2. Armando, A., Mantovani, J., and Platania, L. Bounded Model Checking of C Programs using a SMT solver instead of a SAT solver Technical Report, AI-Lab, DIST, University of Genova, December 19, 2005, 16 pages.

3. Bouquet, F., Dadeau, F., Legeard, B. and Utting, M: JML-Testing-Tools: a Symbolic Animator for JML Specifications using CLP. Procs of the 11th Int. Conf. on Tools and Algorithms for the Construction and Analysis of Systems, Tool session (TACAS'05). Springer-Verlag. LNCS 3440,pp. 551-556, 2005.

4. Clarke E., Kroening D., Sharygina N., Yorav K. : SATABS: SAT-Based Predicate Abstraction for ANSI-C. Procs of the 11th Int. Conf. on Tools and Algorithms for the Construction and Analysis of Systems, Tool session (TACAS'05). SpringerVerlag. LNCS 3440,pp. 570-574, 2005.

5. Ganzinger,H., Hagen,G., Nieuwenhuis, R.,Oliveras, A., and C. Tinelli: DPLL(T): Fast Decision Procedures. Proc. of CAV 2004. Springer-Verlag. LNCS 3114,pp. 175-188, 2004.

6. Gotlieb A., Botella B. and Rueher M : Automatic Test Data Generation using Constraint Solving Techniques. Proc. ISSTA 98, ACM SIGSOFT, vol. 2, pp. 5362, 1998.

7. Leconte M. and Berstel B. : Extending a CP Solver with Congruences as Domains for Program Verification. Procs of CSTVA06.

8. Collavizza H. and Rueher M. : Software Verification using Constraint Programming Techniques. Procs of TACAS 2006, LNCS 3920: 182-196.

9. Cytron R., Ferrante J., Rosen B., Wegman M., and Zadeck K. : Efficently Computing Static Single Assignment Form and the Control Dependence Graph. Transactions on Programming Languages and Systems, 13(4):451-490, October 1991.

10. Ganai M., Gupta A., Ashar P.: DiVer: SAT-Based Model Checking Platform for Verifying Large Scale Systems. Procs of the 11th Int. Conf. on Tools and Algorithms for the Construction and Analysis of Systems, Tool session (TACAS'05). SpringerVerlag. LNCS 3440,pp. 575-580, 2005.

11. M. Moskewicz, C. Madigan, Y. Zhao, L. Zhang, and S. Malik,M: Chaff: Engineering an Efficient SAT Solver. Proc of DAC, pp. 530-535, 2001

12. Robert Nieuwenhuis, Albert Oliveras and Cesare Tinelli. Solving SAT an SAT Modulo Theories: from an Abstract Davis-Putnam-Logemann-Loveland Procedure to DPLL(T). Journal of the ACM (to appear).

13. Sy N.T. and Deville Y.: Automatic test data generation for programs with integer and float variables. Proc of. 16th IEEE International Conference on Automated Software Engineering(ASE01), 2001.

\footnotetext{
${ }^{4}$ see for instance [7] where congruence domains are introduced.
} 